\title{
Thermodynamic Approach to Enhanced Dispersion and Physical Properties in a Carbon Nanotube/Polypeptide Nanocomposite
}

\author{
Conrad Lovell ${ }^{1}$, Kristopher E.Wise ${ }^{2}$, Jae-Woo Kim ${ }^{2}$, Peter T. Lillehei ${ }^{3}$, Joycelyn S. \\ Harrison $^{3}$, Cheol Park ${ }^{2}$ \\ ${ }^{1}$ Department of Materials Science and Engineering, University of Virginia, 22904 \\ ${ }^{2}$ National Institute of Aerospace, Hampton, Virginia, 23666 \\ ${ }^{3}$ Advanced Materials and Processing Branch, NASA Langley Research Center, Hampton, Virginia, \\ 23681
}

\begin{abstract}
A high molecular weight synthetic polypeptide has been designed which exhibits favorable interactions with single wall carbon nanotubes (SWCNTs). The enthalpic and entropic penalties of mixing between these two molecules are reduced due to the polypeptide's aromatic sidechains and helical secondary structure, respectively. These enhanced interactions result in a well dispersed SWCNT/Poly $\left({ }^{\mathrm{L}}\right.$ Leucine-ran- ${ }^{\mathrm{L}}$-Phenylalanine) nanocomposite with enhanced mechanical and electrical properties using only shear mixing and sonication. At $0.5 \mathrm{wt} \%$ loading of SWCNT filler, the nanocomposite exhibits simultaneous increases in the Young's modulus, failure strain, and toughness of $8 \%, 120 \%$, and $144 \%$, respectively. At one $\mathrm{kHz}$, the same nanotube loading level also enhances the dielectric constant from 2.95 to 22.81 , while increasing the conductivity by four orders of magnitude.
\end{abstract}

Keywords: copolypeptide, single wall carbon nanotube (SWCNT), nanotube/polymer interactions, nanotube/polymer nanocomposite

Next generation aerospace applications will demand strong and lightweight materials that offer additional intrinsic functionalities such as electrical conductivity, sensing, or actuation. Incorporation of single wall carbon nanotubes (SWCNTs) into certain high performance polymers yields a lightweight material with dramatically improved mechanical and electrical properties that also exhibits sensing and actuating behavior ${ }^{1,2}$. Achieving these improved properties depends on the SWCNTs being 
well dispersed and stable with respect to reaggregation. Current nanocomposite fabrication techniques include melt mixing ${ }^{3-5}$ or in situ polymerization 1 . The large size and mass of the dispersed nanotubes and low mobility of the matrix polymer result in kinetic barriers that effectively prevent reaggregation over timescales relevant to most applications, provided the nanocomposite is not subjected to any significant thermal or mechanical perturbations. This caveat may make these kinetically trapped and metastable, but ultimately nonequilibrium, nanocomposites unsuitable for aerospace applications in which temperature excursions, cyclic mechanical loadings, and vibration are unavoidable.

Thermodynamic stability of nanocomposites is governed by the free energy difference between the aggregated state and the dispersed (either individually or small bundles) state. The free energy change that occurs during mixing is composed of enthalpic and entropic contributions, $\Delta \mathrm{G}=\Delta \mathrm{H}$ $\mathrm{T} \Delta \mathrm{S}$. If the free energy difference is negative or nearly zero, the dispersed state will be stable. Consider, for example, the case of large, rod-like fillers dispersed in a typical matrix composed of flexible or semiflexible polymer. In the absence of any countervailing enthalpic stabilizing interactions, entropic depletion interactions ${ }^{6-8}$ will tend to drive the system to demix into coexisting rod-rich and polymer-rich phases ${ }^{9}$. We seek to ameliorate this entropic incompatibility by designing a polymer that is similar in diameter and flexibility to SWCNTs, specifically a polypeptide of amino acid monomers that promote an $\alpha$-helical secondary structure.

Achieving a negative enthalpy change upon mixing, without resorting to covalent modification of the SWCNTs, requires either destabilization of the aggregated state or augmentation of the SWCNT-matrix interaction. Mechanisms for aggregate destabilization include Coulombic repulsion, as might result from SWCNT reduction using alkali metal salts ${ }^{10}$, and steric repulsion, typically achieved by coating SWCNTs with surfactants. These approaches are more appropriate for dispersing nanotubes in solution and are not pursued here. SWCNT-matrix interactions can be augmented by functionalizing the polymer with electron donating or withdrawing group ${ }^{11-12}$. Unfortunately, no 
naturally occurring amino acids contain strong donor or acceptor moities. Dispersion (London) interactions also contribute to the enthalpic term. In particular, aromatic ring systems interact strongly with carbon nanotubes ${ }^{13}$, presumably due to the polarizable $\pi$ systems on both the aromatic functional groups and the nanotube ${ }^{14}$.

With the preceding discussion in mind, we have designed a rod-like, high molecular weight synthetic copolypeptide intended to optimize the enthalpic and entropic contributions to the free energy simultaneously ${ }^{15}$. Phenylalanine (F) was selected for its aromatic side chain, while Leucine (L) was utilized as a comonomer to promote chain helicity ${ }^{16}$, and to increase the solubility and processability of polyphenylalanine. In what follows, we describe the preparation and characterization of SWCNT/polyLF nanocomposites and demonstrate their excellent stability and material properties.

The SWCNT/polyLF Young's moduli as a function of carbon nanotube loading are summarized in Table 1 and plotted in Figure 1(a). The Young's modulus increases steadily with increasing SWCNT content up to a loading of $0.75 \mathrm{wt} \%$ where it reaches $3.09 \mathrm{GPa}$, an increase of $\sim 15 \%$ over the pristine sample $(2.70 \mathrm{GPa})$. Further increase of the SWCNT content to $2.0 \mathrm{wt} \%$ yields a small additional improvement, with the modulus increasing to $3.23 \mathrm{GPa}$, or a $\sim 20 \%$ increase over the pure polymer. The ultimate tensile strengths (UTS) of the SWCNT/polyLF nanocomposites, shown in Figure 1(b), are relatively unaffected by SWCNT incorporation, at least up to loading levels of $2.0 \mathrm{wt}$ $\%$.

While the failure strains of most polymers decrease ${ }^{17-20}$, sometimes drastically, upon addition of reinforcing fillers, our SWCNT/polyLF nanocomposites actually show an increase in this material property. Starting from a relatively moderate failure strain of $7 \%$ for the pure polypeptide in Figure 1(c), the addition of SWCNTs results in a maximal increase of $121 \%$ at $0.5 \mathrm{wt} \%$ loading and a still significant gain of $90 \%$ at a loading level of $2.0 \mathrm{wt} \%$. Figure $1(\mathrm{~d})$ plots toughness, which is related to failure strain, as a function of SWCNT loading. Toughness, the energy required to break a material, is estimated by measuring the work of rupture (WOR), or the area beneath a load versus displacement 
curve, and dividing this by the specimen's cross-sectional area. Polymers typically become more brittle with the addition of reinforcing fillers $17^{-181920}$, but this copolypeptide's toughness actually improves with addition of carbon nanotubes, becoming more ductile while simultaneously increasing its modulus and maintaining its overall strength. As shown in Figure 1(d), the pristine sample exhibited a toughness of $83.18 \mathrm{~kJ} / \mathrm{m}^{2}$. This toughness value increases by $144 \%$ for the $0.5 \mathrm{wt} \%$ SWCNT loading, and $116 \%$ for the $2.0 \mathrm{wt} \%$ sample. Note that this data was not obtained with a typical Impact Test (e.g. Charpy Test), and is merely a relative measure for comparison among the samples in this study. While the data exhibits a high standard deviation, the increase in the composite's toughness can not be disregarded, particularly at 0.5 and $0.75 \mathrm{wt} \%$, where the toughness values are well above the pristine sample.

The frequency dependent $\mathrm{AC}$ conductivities of each nanocomposite sample are plotted in Figure 2(a). The traces exhibit increasingly metallic (i.e. frequency independent) behavior with higher loading levels of SWCNTs, attaining an essentially constant conductivity of $\sim 4.2 \times 10^{-5} \mathrm{~S} / \mathrm{cm}$ at $2.0 \mathrm{wt}$ $\%$. This graph exhibits the prototypical behavior of a conductive filler-insulating matrix composite, passing through a percolation transition at $\sim 0.5 \mathrm{wt} \%$ and reaching a plateau above $0.75 \mathrm{wt} \%$. Figure 2(b) presents the frequency dependence of the permittivities for each of the samples except for the 2.0 $\mathrm{wt} \%$ sample, which was too conductive to measure. Insulating materials exhibit an essentially constant permittivity across the frequency range, as observed for the pure polymer and the 0.075 and $0.2 \mathrm{wt} \%$ samples. Once percolation is achieved, however, we find enormous increases in the low frequency permittivities, as expected for materials exhibiting conductive behavior. Interfacial polarization at the surface of the SWCNTs is responsible for this increase at low frequencies and a similar observation has been reported elsewhere ${ }^{21,22}$. An order of magnitude increase in the permittivity is observed between the pure polymer (2.95) and the $0.75 \mathrm{wt} \%$ sample $(35.48)$ at $1 \mathrm{kHz}$. This is consistent with the percolation transition determined from the electrical conductivity measurements. To quantify the value of the 
percolation threshold, we show in Figure 2(c) a percolation graph with the expected power law behavior. This equation is given by

$$
\sigma_{c}=\sigma_{0}\left(v-v_{c}\right)^{t}
$$

where $\sigma_{\mathrm{c}}$ is the composite conductivity, $\sigma_{0}$ is a pre-exponential factor that depends on the filler conductivity, $v$ is the SWCNT volume fraction, $v_{c}$ is the concentration at the percolation threshold, and $t$ is the conductivity critical exponent. By fitting the percolation equation to the $0.01 \mathrm{~Hz}$ experimental data (to approximate DC conductivities), we obtain threshold concentration $v_{\mathrm{c}}$ of $0.48 \mathrm{vol} . \%(0.51 \mathrm{wt}$ \%). This value is consistent with the behavior observed in Figures 2(a) and 2(b), where the transition from insulating to conductive behavior occurs very near $0.5 \mathrm{wt} \%$. The remarkable increases in conductivity in this composite ( $\sim 4$ and 7 orders of magnitude at 0.5 and $2.0 \mathrm{wt} \%$, respectively) will permit its utilization in low conductivity applications such as electrostatic charge dissipation.

The mechanical and electrical results for the SWCNT/polyLF nanocomposites clearly show enormous gains with the addition of the nanotube fillers. The enhanced interaction between the rodlike polyLF matrix and the filler, due to the optimization of the enthalpic and entropic contributions to the free energy of mixing, results in superior dispersion and improved energy transfer across the matrix/filler interface. To visualize the carbon nanotube dispersion, scanning electron micrographs of the SWCNT/polyLF nanocomposite film surfaces were taken, shown in Figures 3(a) and (b). These images, representing the 0.5 and $2.0 \mathrm{wt} \%$ samples respectively, were taken at an accelerating voltage between $20-30 \mathrm{kV}$. The high voltages used in these micrographs create a beam that penetrates through the polymer matrix. These "poly-transparent" images give better insight into the level of dispersion by revealing nanotube bundles embedded deep into the nanocomposite. These bundles are not visible in conventional topographical images ${ }^{23}$. These poly-transparent images reveal flexible nanotube networks throughout the matrix with a few loosely entangled nanotube agglomerates. Overall, Figures 3(a) and 
(b) show that reasonably uniform carbon nanotube dispersion was achieved in polyLF using only shear mixing and sonication (i.e. without resorting to surfactants or covalent functionalization).

In Figures 3(c) - (f), the fractured cross-section of the $0 \mathrm{wt} \%$ (Figures 3(c) and (d)) and $2.0 \mathrm{wt}$ $\%$ (Figures 3(e) and (f)) samples are shown. Carbon nanotubes can clearly be seen as thin, bright filaments in images 3(e) and (f), a result of the emission of secondary electrons. The $0 \mathrm{wt} \%$ sample images show porous fibrous morphology with thicker protrusions and connections between polymer regions. Many of these connections were broken during the fracturing process, as shown in Figure 3(d). In contrast, the $2.0 \mathrm{wt} \%$ sample exhibits much finer fibrillar structures, indicated by the thin, bright regions in the images. Also note that the SWCNTs clearly span the crevices of the nanocomposite, giving these images a threadlike, connected, and rough appearance compared to images of the pure polymer. This increased roughness and persistent spanning of the crevices suggest the mechanism for the nanocomposite's enhanced toughness.

To relate the filler dispersion found in the SEM images with the mechanical reinforcement data, and to gain better insight on the SWCNT/polyLF Young's modulus trend, we compare the experimental data to the predictions of a modified Cox $\mathrm{Model}^{24}$. This model builds upon the original Cox model, which is a variation on the rule of mixtures. The composite modulus is expressed as an additive combination of the fiber and matrix contributions:

$$
E_{C}=E_{F} V_{F} f\left(l_{F}\right) C_{0}+E_{M}\left(1-V_{F}\right)
$$

where $E_{\mathrm{C}}, E_{\mathrm{F}}, E_{\mathrm{M}}$ are the Young's Moduli of the composite, fiber and matrix, respectively, and $V_{\mathrm{F}}$ is the volume fraction of the carbon nanotubes. The $C_{0}$ term is added to allow for fiber alignments other than uniaxial, which is the only arrangement considered in the simple rule of mixtures. In the limit of $f\left(l_{\mathrm{F}}\right)$ 
and $C_{0}$ equal to unity, the classic rule of mixtures is recovered. The $f\left(l_{\mathrm{F}}\right)$ term is introduced to account for the finite length of the reinforcing fibers and may be written as:

$$
\begin{gathered}
f\left(l_{F}\right)=1-\frac{\tanh \left(\frac{\beta * l_{F}}{2}\right)}{\left(\frac{\beta * l_{F}}{2}\right)} \\
\text { where, } \beta^{r}=\frac{r G_{M}}{E_{F} r_{F}^{r} \ln \left(\frac{R}{r_{F}}\right)}
\end{gathered}
$$

In this expression, $G_{\mathrm{M}}$ is the matrix shear modulus, $r_{\mathrm{F}}$ is the radius of the filler fiber, and $R$ is the radius of the effective fiber, which includes both the filler and the region of matrix surrounding the filler for which the mechanical properties are substantially different from the bulk matrix. For a hexagonally packed arrangement of the fibers, $R / r_{\mathrm{F}}$ equals $\left[\left(2 \pi /\left(\sqrt{ } 3^{*} \mathrm{~V}_{\mathrm{F}}\right)\right)^{1 / 2}\right]^{25}$. The experimental data in Figure 4 have been normalized to the modulus of the pristine copolypeptide and the filler concentrations have been converted to vol. $\%$ by assuming densities of $1.23 \mathrm{~g} / \mathrm{cm}^{3}$ for polyLF ${ }^{26}$ (averaging the densities of the two amino acids) and $1.35 \mathrm{~g} / \mathrm{cm}^{3}$ for SWCNTs ${ }^{27}$. The calculated values in Figure 4 were obtained with the following parameters: a polyLF Poisson's ratio of 0.4 , a SWCNT modulus of $1 \mathrm{TPa}$, a fiber orientation factor $\mathrm{C}_{90}=0.33$ (isotropic orientation24), a SWCNT bundle length of $1 \mu \mathrm{m}$, and a SWCNT bundle diameter of $32 \mathrm{~nm}$. These are all typical values for polymers and carbon nanotubes, and the bundle dimensions were estimated from HRSEM images of pure SWCNTs and nanocomposite crosssectional images. Figure 4 shows that the model predictions are in good agreement with the experimental data up to the $0.75 \mathrm{wt} \%$ (0.69 vol. \%) sample. At $2.0 \mathrm{wt} \%$ (1.83 vol. \%), however, the experimental Young's modulus does not exhibit the continued increase in modulus predicted by the current model. The failure of the model to predict the modulus at higher loadings could be due to its 
assumption that the level of dispersion is preserved at higher loadings. The presence of small bundles of overlapping carbon nanotubes at higher volume fractions would result in a sublinear increase of surface area with respect to volume fraction. As the interfacial interaction of the SWCNTs with the matrix depends directly on the exposed surface area, the model would tend to over-predict the modulus as bundling increases.

In order to investigate the origin of the increased polyLF toughness, we performed in-situ tensile tests in a JEOL 5600 JSM-SEM fitted with a Gatan Microtest 200 stage and Deben controller. An in-situ load-displacement curve for a $2.0 \mathrm{wt} \% \mathrm{SWCNT} / \mathrm{LF}$ sample performed inside of the SEM chamber is shown in Figure 5(a). Figures 5(b) and (c) show consecutive SEM images of one location obtained just after yielding, and after extensive deformation, respectively, which correspond to the (b) and (c) markers on the load displacement curve in Figure 5(a). The load drops at (b) and (c) in this Figure indicate a stress relaxation which occurred while holding the strain constant during imaging. The progressive initiation and slow propagation of many small cracks shown in Figure 5(b) and (c), as opposed to one catastrophic crack in brittle failure, at least partially accounts for the high energy absorption and toughness that this material exhibits. In general, crack fronts can change in length as they interact with inhomogeneous inclusions. Many nanotubes in the percolated network throughout the matrix can interact with the crack fronts, absorbing large amounts of energy and toughening the composite. In the close-up image shown in Figure 5(d), it is apparent that small cracks are held together by the SWCNTs and this bridging is likely to be one of the major toughness-improving mechanisms. This spanning by carbon nanotubes can also be seen clearly in the cross-sectional images of Figure 3(e) and (f) discussed earlier. The strong, flexible, and ductile SWCNTs act like a thermoplastic toughener by bridging cracks and exerting compressive traction in the crack wake ${ }^{28}$. The SWCNTs are pulled out during the crack opening process, absorbing significant amounts of energy due to the large interfacial area between the SWCNTs and the matrix. Carbon nanotubes, which have extremely large aspect ratios, are particularly effective for crack bridging, as this mechanism tends to 
favor larger fillers ${ }^{29}$. Better dispersion and more interfacial bonding, which occur between our specifically designed copolypeptide and SWCNTs, can provide higher energy absorption resulting in enhanced toughness. Another mechanism for increasing the work of rupture is effective crack path deflection by the uniformly dispersed nanotubes, as in Figure 5(e). This deflection changes the crack propagation from a mode-I (tensile) to mode-II (shear) failure character, which enhances energy absorption as most materials are more resistant to this latter type of crack opening. Other obvious contributors to the improved toughness are physical entanglements of nanotubes and nanotube bundle bifurcation/splitting as shown in Figure 3(f). Ubiquitous physical entanglements and bifurcated/split nanotubes have been reported before ${ }^{30}$. This characteristic enables good energy absorption as a result of the excellent binding forces between the nanotubes in the bundles during fracture. In-situ SEM images of the fractured nanocomposite shown in Figure 5 substantiate the toughness results discussed above, and illustrate how excellent dispersion and enhanced compatibility between the matrix and filler provide a more mechanically robust composite for various sensing and actuation applications.

In conclusion, nanocomposites consisting of a Leucine-Phenylalanine copolypeptide and single wall carbon nanotubes were developed by a simple mixing and sonicating technique. This particular copolypeptide was designed to provide both a favorable enthalpic stabilization from the presence of aromatic sidechains, and to minimize the unfavorable entropic contributions due to its helical secondary structure. The improved compatibility achieved in this system results in excellent filler dispersion in the matrix, as demonstrated in the scanning electron micrographs. Furthermore, it leads to a remarkable increase in many of the copolypeptide's physical properties, including elastic modulus, failure strain, toughness, conductivity, and dielectric constant. The increases in failure strain and toughness are perhaps the most intriguing, considering that polymers with reinforcing fillers typically show reductions in both properties. These results show that carbon nanotubes can provide enhanced durability and conductivity to many of the otherwise fragile copolypeptides which may be needed for harsh sensing or actuation applications. 


\section{Methods}

The Poly ( ${ }^{\mathrm{L}}$-Leucine-ran- $\left.{ }^{\mathrm{L}}-\mathrm{Phenylalanine}\right)$, or polyLF, was provided by the University of California, Santa Barbara ${ }^{31}$. Purified SWCNTs made by the High Pressure CO (HiPCO) synthesis method were purchased from Carbon Nanotechnologies, Inc. Chloroform from Fisher Scientific was used to process the copolypeptide. All materials were used as received.

Six SWCNT/LF samples were made with SWCNT loadings of $0,0.075,0.2,0.5,0.75$, and 2.0 wt $\%$. For the samples containing carbon nanotubes, a suspension of chloroform and SWCNTs was first made and pulse-sonicated (5 seconds on, 5 seconds off) with a cup sonicator for 18 minutes at 12 $\mathrm{W}$ and $20 \mathrm{kHz}$, and then appropriate amounts were added to the dry copolypeptide powder. A low wattage was used to sonicate the nanotube solutions to avoid SWCNT damage. More chloroform was then added to lower the polymer concentration to $4 \mathrm{wt} \%$. Every polymer solution (with or without SWCNTs) was also pulse-sonicated ( 5 seconds on, 5 seconds off) for 18 minutes in a cup sonicator (36 $\mathrm{W}, 20 \mathrm{kHz})$, followed by one hour of sonication in a bath sonicator $(70 \mathrm{~W}, 42 \mathrm{kHz})$. All solutions were stirred constantly, except when subjected to cup sonication. Low molecular weight surfactants were not used to aid carbon nanotube dispersion due to their tendency to act as plasticizers, which may degrade the physical properties of the polymer matrix. Furthermore, functionalization of the nanotubes was not used since this damages the $\mathrm{sp}^{2}$ bonding of the nanotubes ${ }^{32}$, ultimately diminishing the properties of the copolypeptide composites as well. After the samples were sonicated and stirred, they were cast onto a Teflon ${ }^{\circledR}$ substrate using a film applicator. Following overnight solvent evaporation in a dessicator, the samples were placed in a vacuum oven for another 48 hours at room temperature to ensure complete solvent removal. The composite films, approximately 30-35 $\mu \mathrm{m}$ thick, detached easily from the substrate for characterization.

A Hitachi S-5200 high resolution scanning electron microscope (HRSEM), with a field emission electron gun and in-lens detector, was used to examine nanotube dispersion near the surface 
of the samples, as well as the cross-sectional sample morphology. SEM samples of the SWCNT/polyLF composite films for surface imaging were cut to approximately $6 \mathrm{~mm} \times 3 \mathrm{~mm}$ and mounted on sample stages with silver paste. Cross-sectional SEM samples were fractured in liquid nitrogen, and samples below the electrical percolation threshold $(<0.47$ vol\%) were coated with 10 nanometers of silver. A JEOL 5600 JSM-SEM, equipped with a Gatan Microtest 200 stage and Deben controller, was used for in-situ fracture imaging. Tensile properties of the films were evaluated at room temperature using an Instron 5848 MicroTester equipped with a 1000 Newton load cell. Instron samples were cut in three mm wide rectangular strips using a JDC precision sample cutter (ThwingAlbert Instrument Company), and tested with a $30 \mathrm{~mm}$ gauge length at an extension rate of three $\mathrm{mm} / \mathrm{min}$ according to ASTM 882. Six samples were tested for each composite film. A Novocontrol broadband dielectric converter and a Solartron SI1260 impedance gain/phase analyzer were used to evaluate electrical and dielectric properties. A $25.4 \mathrm{~mm}$ diameter silver electrode (approximately 50 nm thick) was deposited on both sides of the sample prior to testing.

\section{Acknowledgements}

The authors thank Nancy M. Holloway for electroding the samples. They also acknowledge Timothy Deming, Galen Stucky and Edward Worthington for supplying the LF copolypeptide. Lovell appreciates the guidance and discussion of Dr. James M. Fitz-Gerald at the University of Virginia. Park and Wise appreciates NASA University Research, Engineering and Technology Institute on Bio Inspired Materials (BIMat) under Award No. NCC-1-02037 for support in part. 


\section{Figure Legends}

Figure 1. (a) Young's modulus, (b) ultimate tensile strength (UTS), (c) failure strain, and (d) toughness of the SWCNT/polyLF nanocomposite at the various SWCNT loadings.

Figure 2. The SWCNT/polyLF nanocomposite's (a) conductivity versus frequency, and (b) dielectric constant versus frequency. Image (c) shows a power law plot of the SWCNT/polyLF nanocomposite's conductivities assuming a critical SWCNT volume fraction (percolation threshold) of $0.48 \%$.

Figure 3. HRSEM images of the SWCNT/polyLF nanocomposite: (a) $0.5 \mathrm{wt} \%$ surface (b) $2.0 \mathrm{wt} \%$ surface, $(\mathrm{c}, \mathrm{d}) 0 \%$ cross-section and $(\mathrm{e}, \mathrm{f}) 2.0 \mathrm{wt} \%$ cross-section. The cross-sectional image samples were fractured in liquid nitrogen, with the $0 \mathrm{wt} \%$ images coated with 10 nanometers of silver to minimize charging.

Figure 4. Normalized Young's Modulus versus SWCNT volume percent (data fitted to a modified Cox Model24) of the SWCNT/polyLF nanocomposite.

Figure 5. In-situ fracturing of the $2.0 \mathrm{wt} \% \mathrm{SWCNT} /$ polyLF sample showing (a) the load versus displacement curve, (b) an image just after yielding, and (c) an image after extensive deformation. Higher magnification images show (d) nanotube bridging and (e) crack path deflection.

Table 1. Values of several SWCNT/polyLF nanocomposite properties.

\begin{tabular}{|c|c|c|c|c|c|c|}
\hline $\begin{array}{l}\text { SWCNT } \\
\text { wt } \%\end{array}$ & $\begin{array}{l}\text { Modulus } \\
\text { (GPa) }\end{array}$ & $\begin{array}{c}\text { UTS } \\
\text { (MPa } \\
\text { ) }\end{array}$ & $\begin{array}{c}\text { Failure Strain } \\
(\%)\end{array}$ & $\begin{array}{c}\text { Toughness } \\
\left(\mathbf{k J} / \mathbf{m}^{2}\right)\end{array}$ & $\begin{array}{l}\log (\sigma) @ \\
1 \mathrm{kHz}(\mathrm{S} / \mathrm{cm})\end{array}$ & $\underset{\mathbf{k H z}}{\varepsilon @ 1}$ \\
\hline 0 & 2.70 & 48.75 & 7.02 & 83.18 & -11.69 & 2.95 \\
\hline 0.075 & 2.71 & 46.35 & 10.02 & 121.24 & -11.44 & 3.22 \\
\hline 0.2 & 2.84 & 45.30 & 14.11 & 172.05 & -11.25 & 3.72 \\
\hline 0.5 & 2.94 & 48.41 & 15.51 & 203.22 & -7.81 & 22.81 \\
\hline 0.75 & 3.09 & 53.51 & 12.08 & 177.48 & -5.88 & 38.48 \\
\hline 2.0 & 3.23 & 49.51 & 13.31 & 179.26 & -4.38 & - \\
\hline
\end{tabular}


(a)

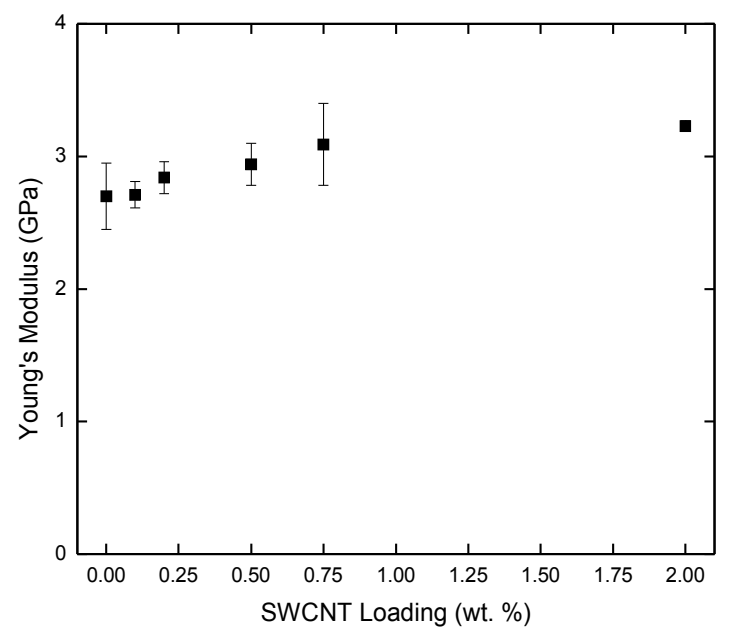

(c)

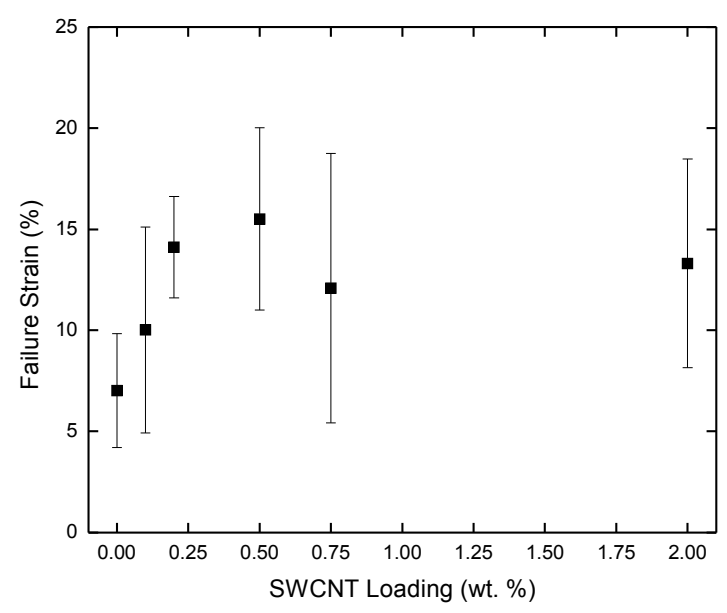

(b)

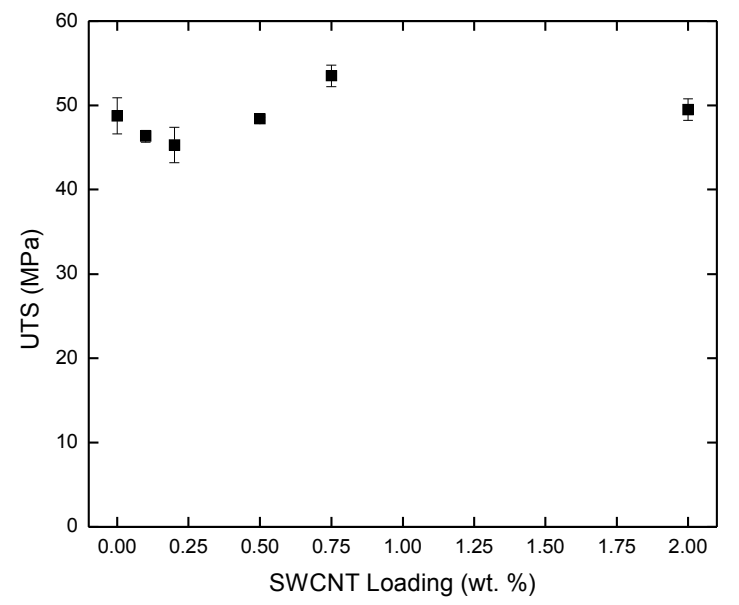

(d)

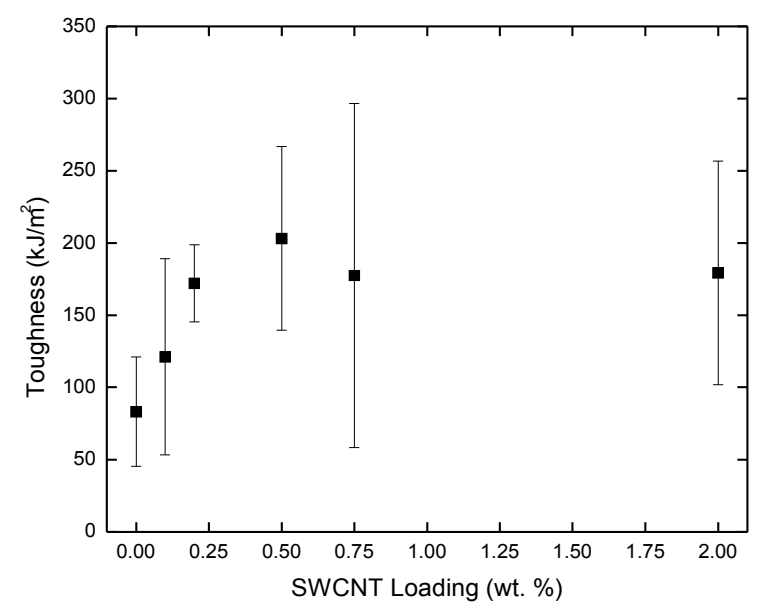

Figure 1. (a) Young's modulus, (b) ultimate tensile strength (UTS), (c) failure strain, and (d) toughness of the SWCNT/polyLF nanocomposite at the various SWCNT loadings.

C. Lovell, K. E. Wise, J.-W. Kim, P. T. Lillehei, and C. Park 
(a)

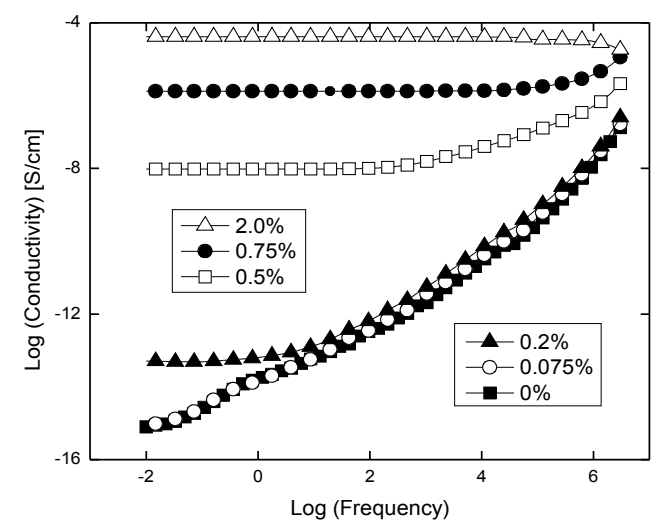

(b)

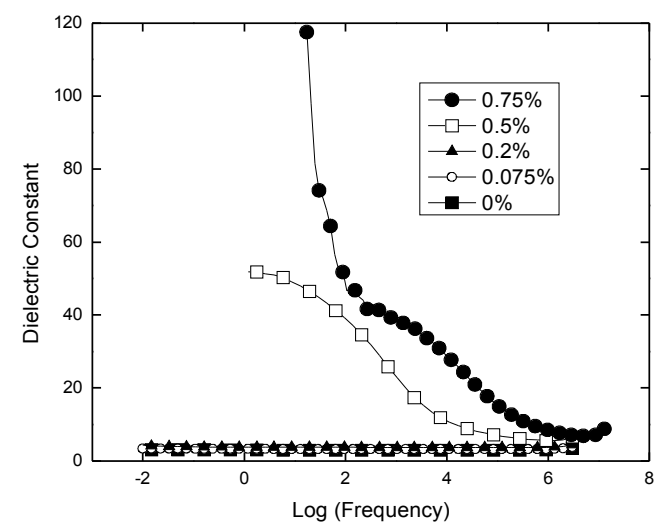

(c)

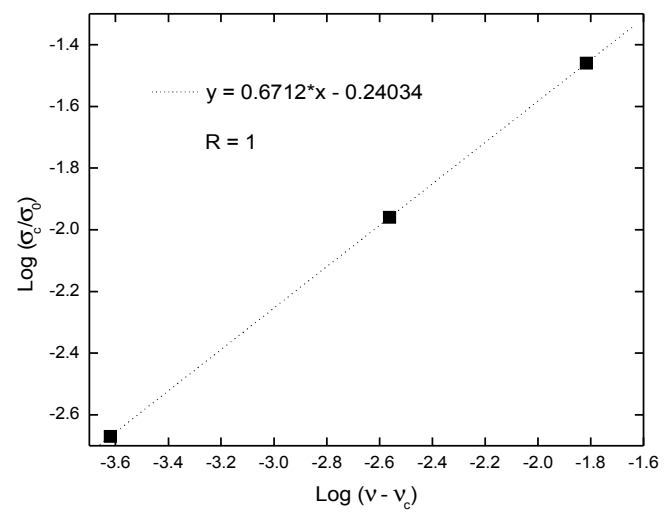

Figure 2. The SWCNT/polyLF nanocomposite's (a) conductivity versus frequency, and (b) dielectric constant versus frequency. Image (c) shows a power law plot of the SWCNT/polyLF nanocomposite's conductivities assuming a critical SWCNT volume fraction (percolation threshold) of $0.48 \%$.

C. Lovell, K. E. Wise, J.-W. Kim, P. T. Lillehei, and C. Park 

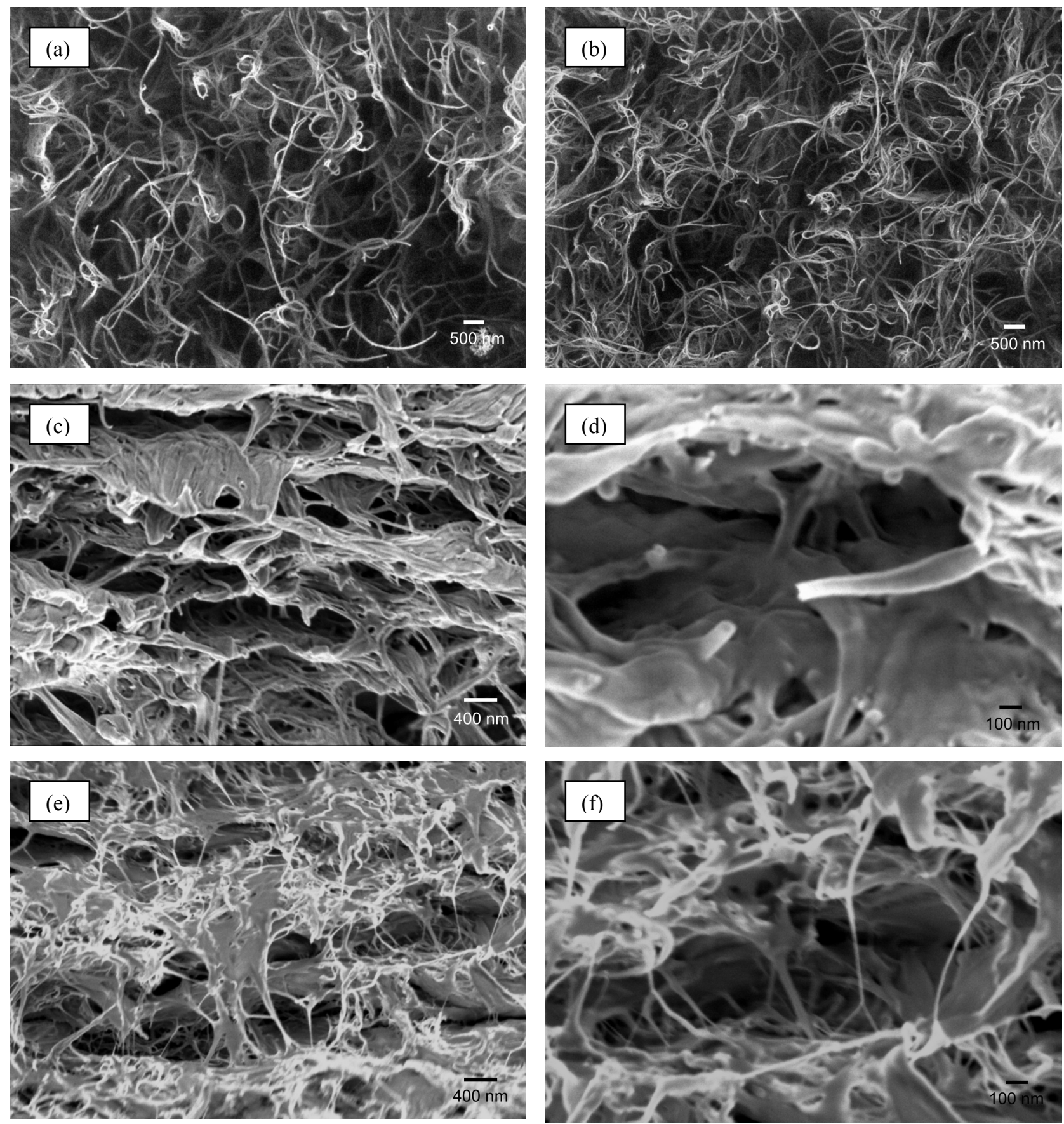

Figure 3. HRSEM images of the SWCNT/polyLF nanocomposite: (a) $0.5 \mathrm{wt} \%$ surface (b) $2.0 \mathrm{wt} \%$ surface, $(\mathrm{c}, \mathrm{d}) 0 \%$ cross-section and $(\mathrm{e}, \mathrm{f}) 2.0 \mathrm{wt} \%$ cross-section. The cross-sectional image samples were fractured in liquid nitrogen, with the $0 \mathrm{wt} \%$ images coated with 10 nanometers of silver to minimize charging.

C. Lovell, K. E. Wise, J.-W. Kim, P. T. Lillehei, and C. Park 


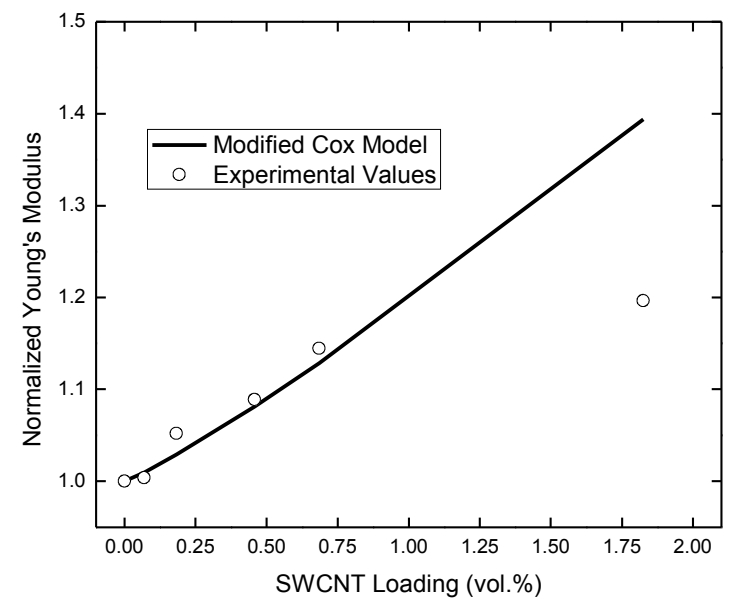

Figure 4. Normalized Young's Modulus versus SWCNT volume percent (data fitted to a modified Cox Model24) of the SWCNT/polyLF nanocomposite.

C. Lovell, K. E. Wise, J.-W. Kim, P. T. Lillehei, and C. Park 
(a)

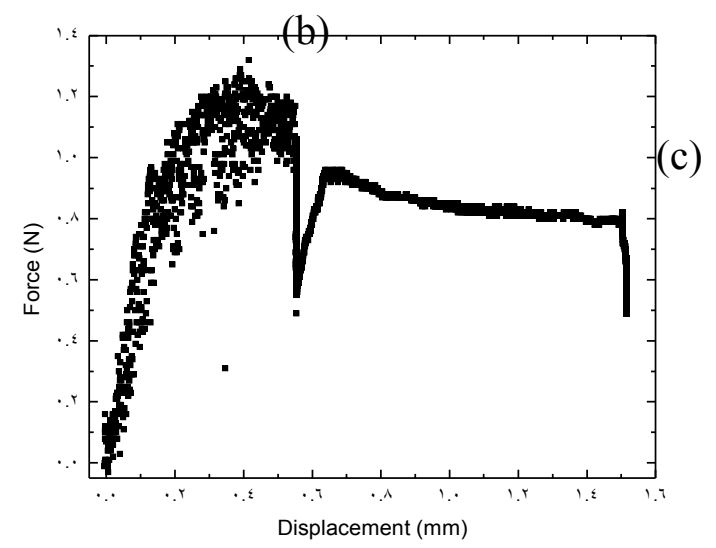

(b)

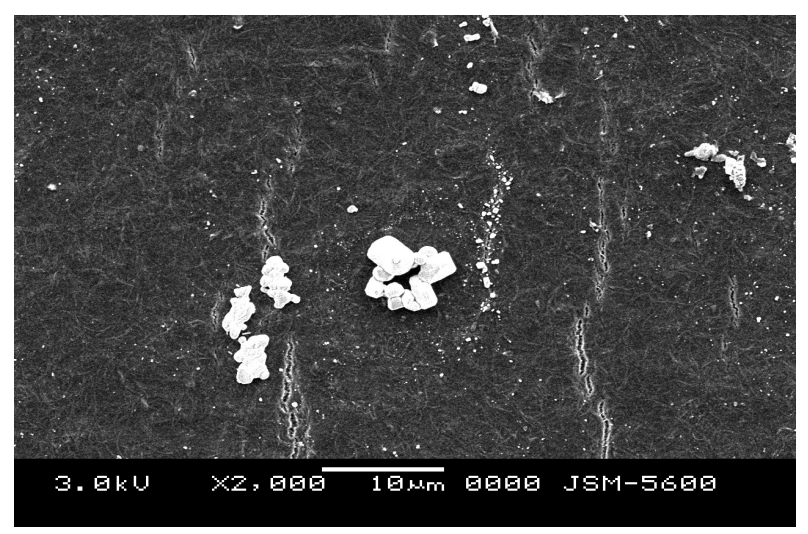

(d)

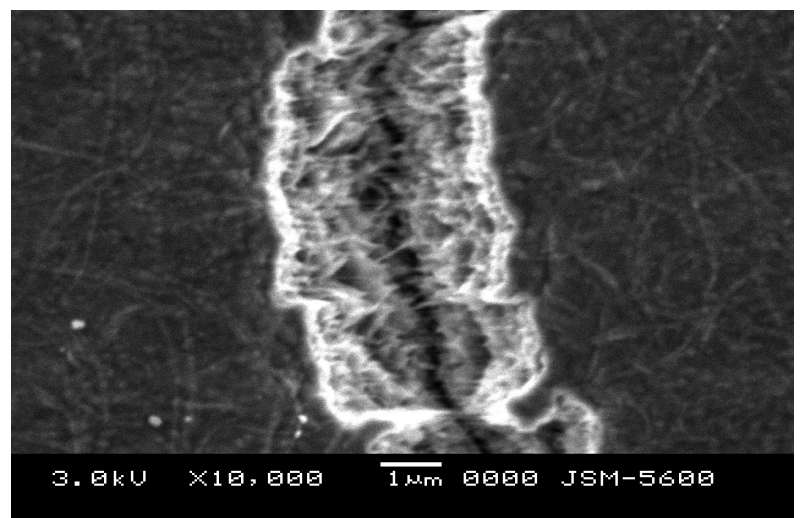

(c)

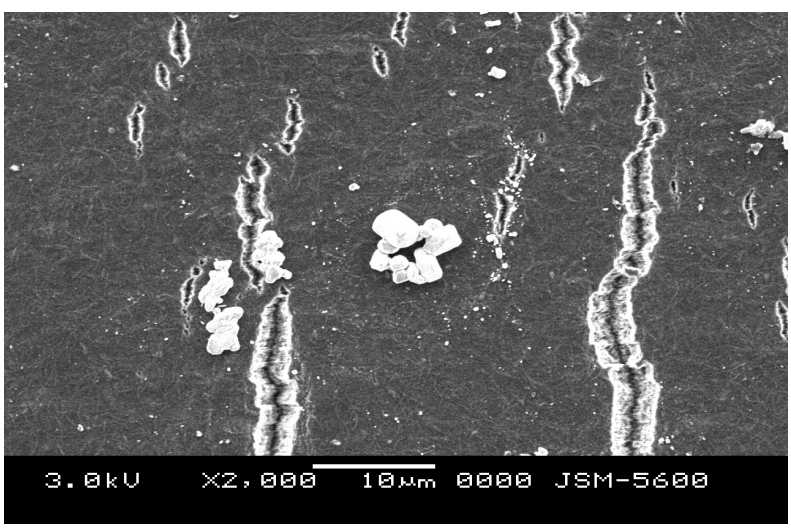

(e)

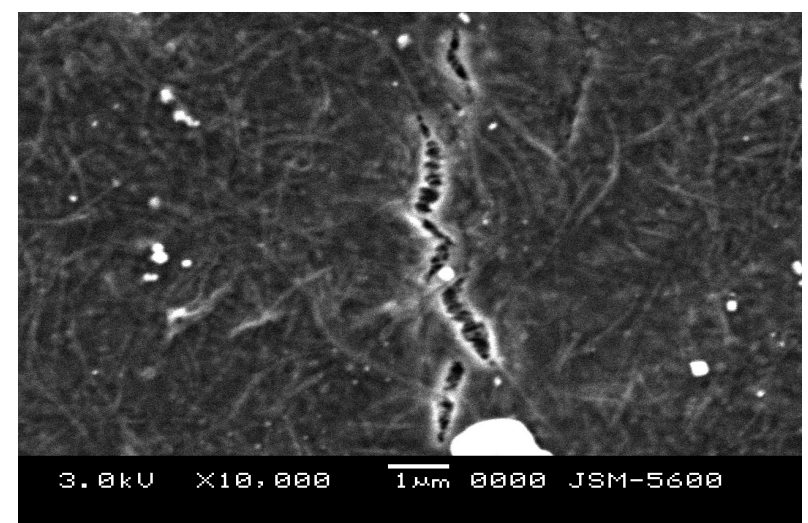

Figure 5. In-situ fracturing of the $2.0 \mathrm{wt} \% \mathrm{SWCNT} /$ polyLF sample showing (a) the load versus displacement curve, (b) an image just after yielding, and (c) an image after extensive deformation. Higher magnification images show (d) nanotube bridging and (e) crack path deflection.

C. Lovell, K. E. Wise, J.-W. Kim, P. T. Lillehei, and C. Park

\section{References}


${ }^{1}$ Park, C. et. al. Dispersion of single wall carbon nanotubes by in situ polymerization under sonication. Chem. Phys. Lett. 364, 303-308 (2002).

${ }^{2}$ Breuer, O. \& Sundararaj, U. Big returns from small fibers: A review of polymer/carbon nanotube composites. Polym. Composite. 25, 630-645 (2004).

${ }^{3}$ Haggenmueller, R., Gommans, H. H., Rinzler, J. E., Fischer, J. E., \& Winey, K. I. Aligned single-wall carbon nanotubes in composites by melt processing methods. Chem. Phys. Lett. 330, 219-225 (2000).

${ }^{5}$ Moniruzzaman, M., \& Winey, K. I. Polymer nanocomposites containing carbon nanotubes. Macromolecules 39, 5194-5205 (2006).

${ }^{6}$ Asakura, S., \& Oosawa, F. On interaction between two bodies immersed in a solution of macromolecules. J. Chem. Phys. 22, 1255-1256 (1954).

${ }^{9}$ More extensive discussions of the difficulties of preparing stable nanocomposites filled with carbon nanotubes [31-34] and other rod-like polymers [36-43] are available in the literature.

${ }^{10}$ Petit, P., Derre, A., Anglaret, E., Poulin, P., \& Penicaud, A. Spontaneous dissolution of a single-wall carbon nanotube salt. J. Am. Chem. Soc. 127, 8-9 (2005).

${ }^{11}$ Wise, K. E., Park, C., Siochi, E. J., \& Harrison, J. S. Stable dispersion of single wall carbon nanotubes in polyimide: the role of noncovalent interactions. Chem. Phys. Lett. 391, 207-211 (2004).

${ }^{12}$ Poenitzsch, V. Z. et. al. Effect of electron-donating and electron-withdrawing groups on peptide/single-walled carbon nanotube interactions. J. Am. Chem. Soc. 129, 14724-14732 (2007).

${ }^{13}$ Chen, R. J., Zhang, Y., Wang, D., \& Dai, H. Noncovalent sidewall functionalization of single-walled carbon nanotubes for protein immobilization. J. Am. Chem. Soc. 123, 3838-3839 (2001).

${ }^{14}$ Grimme, S. Do special noncovalent pi-pi stacking interactions really exist? Angew. Chem. Int. Ed. 47, 3430-3434 (2008). In accordance with the conclusions reached by Grimme, we avoid using the ambiguous term "pi-pi interaction" to describe the interaction between the phenyl ring of phenylalanine and the SWCNT. We also note that we do not currently have any experimental evidence proving that the phenyl ring lies in a stacked geometrical configuration with respect to the nanotube, although preliminary modeling results do suggest that this is the case.

${ }^{15}$ Polypeptides can be tailored to optimize their interactions with carbon nanotubes by judiciously choosing the appropriate amino acid sequence. See for example refs [12,44-49].

${ }^{16}$ Nygren, P., Lundqvist, M., Broo, K., and Jonsson, B.-H. Fundamental design principles that guide induction of helix upon formation of stable-peptide-nanoparticle complexes. Nano Lett. 8, 1844-1852 (2008).

${ }^{17}$ Wang, S.-F., Shen, L., Zhang, W.-D., \& Tong, Y.-J. Preparation and mechanical properties of chitosan/carbon nanotubes composites. Biomacromolecules 6, 3067-3072 (2005).

${ }^{21}$ Kim, B., Lee, J., \& Yu, I. Electrical properties of single-wall carbon nanotube and epoxy composites. J. Appl. Phys. 94, 6724-6728 (2003).

${ }^{22}$ Park, C., Kang, J.H., Harrison, J.S., Costen, R.C., \& Lowther, S.E. Actuating single wall carbon nanotube-polymer composites: intrinsic unimorphs. Adv. Mater. 20, 2074-2079 (2008).

${ }^{23}$ Kovacs, J.Z. et. al. Analyzing the quality of carbon nanotube dispersions in polymers using scanning electron microscopy. Carbon 45, 1279-1288 (2007).

${ }^{24}$ Kelly, A. \& Zweben, C. (eds.) Comprehensive Composite Materials (Elsevier Science, Amsterdam, 2000).

${ }^{25} \mathrm{Fu}$, S.-Y., \& Lauke, B. The elastic modulus of misaligned short-fiber-reinforced polymers. Compos. Sci. Technol. 58, 389-400 (1998). 
${ }^{26}$ Weast, R.C. (ed) CRC Handbook of Chemistry and Physics (CRC Press, Cleveland, 1977).

${ }^{27}$ Gao, G., Cagin, T., \& Goddard III, W.A. Energetics, structure, mechanical and vibrational properties of single-walled carbon nanotubes. Nanotechnology 9, 184-191 (1998).

${ }^{28}$ Riew, C.K., \& Kinloch, A.J. Toughened plastics I: science and engineering. American Chemical Society, Washington D.C., 1993.

${ }^{29}$ Sigl, L.S., Mataga, P.A., Dalgleish, B.J., McMeeking, R.M., \& Evans, A.G. On the toughness of brittle materials reinforced with a ductile phase. Acta Metall. 36, 945-953 (1988).

${ }^{30}$ McLachlan, D.S. et. al. AC and DC percolative conductivity of single wall carbon nanotube polymer composites. $J$. Polym. Sci. Pol. Phys. 43, 3273-3287 (2005).

${ }^{31}$ The polyLF was provided by the Deming Group, formerly at the University of California, Santa Barbara, and currently at the University of California, Los Angeles.

${ }^{32}$ Garg, A. \& Sinnott, S.B. Effect of chemical functionalization on the mechanical properties of carbon nanotubes. Chem. Phys. Lett. 295, 273-278 (1998). 